\title{
On a nonlocal problem for parabolic equation with time dependent coefficients
}

\author{
Nguyen Duc Phuong ${ }^{1}$, Ho Duy Binh², Le Dinh Long ${ }^{2}$ and Dang Van Yen ${ }^{3 *}$
}

"Correspondence:
ydangw@yahoo.com.vn
${ }^{3}$ Faculty of Information Technology,
Ho Chi Minh City University of
Technology, Ho Chi Minh City,
Vietnam
Full list of author information is
available at the end of the article

available at the end of the article

\begin{abstract}
This paper is devoted to the study of existence and uniqueness of a mild solution for a parabolic equation with conformable derivative. The nonlocal problem for parabolic equations appears in many various applications, such as physics, biology. The first part of this paper is to consider the well-posedness and regularity of the mild solution. The second one is to investigate the existence by using Banach fixed point theory.
\end{abstract}

MSC: 35R11;35B65; 26A33

Keywords: Parabolic equation; Existence and regularity; Conformable derivative

\section{Introduction}

Let $T$ be a positive number and $\Omega \subset \mathbb{R}^{d}(d \geq 1)$ be a bounded domain with the smooth boundary $\partial \Omega$. In this paper, we consider the nonlocal value problem for parabolic equation as follows:

$$
\begin{cases}\frac{\mathscr{C}_{\partial} \beta}{\partial t^{\beta}} u(x, t)-\psi(t) \Delta u=F(x, t, u(x, t)), & x \in \Omega, t \in(0, T), \\ u(x, t)=0, & x \in \partial \Omega, t \in(0, T), \\ a u(x, 0)+b u(x, T)=\varphi(x), & x \in \Omega,\end{cases}
$$

where the symbol $\frac{\mathscr{C}_{\partial} \beta}{\partial t^{\beta}(t)}$ is called the conformable derivative which is defined clearly in Sect. 2. The function $F$ represents external forces, and the function $\varphi$ is the input datum which will be defined later. The function $\psi$ is called time dependent coefficient.

There are applications of conformable derivative in various models, for example, the harmonic oscillator, the damped oscillator, and the forced oscillator (see [1]), electrical circuits (see [2]), chaotic systems in dynamics (see [3]), quantum mechanics [4]. Based on important notes in the article [5], we observe and think that studying the ODE problem with the compliance derivative is very different from studying the PDE problem with a suitable derivative. The positional results and methods for the ODE and PDEs models are not the same and completely different. In order for the reader to have more access to this kind of fractional diffusion equations with conformable derivative, we refer to [2, 6-17]. In addition, we can find the topics of initial and final problems, which are studied by many authors, in [18-27].

(c) The Author(s) 2021. This article is licensed under a Creative Commons Attribution 4.0 International License, which permits use, sharing, adaptation, distribution and reproduction in any medium or format, as long as you give appropriate credit to the original author(s) and the source, provide a link to the Creative Commons licence, and indicate if changes were made. The images or other third party material in this article are included in the article's Creative Commons licence, unless indicated otherwise in a credit line to the material. If material is not included in the article's Creative Commons licence and your intended use is not permitted by statutory regulation or exceeds the permitted use, you will need to obtain permission directly from the copyright holder. To view a copy of this licence, visit http://creativecommons.org/licenses/by/4.0/. 
Our paper is one of the first results on the nonlocal value problem given with parabolic equations with conformable derivative. In the linear part, we use the techniques of Hilbert scales space. In the nonlinear part, to establish the existence and uniqueness of the solution, we must use the Banach fixed mapping theorem combined with some techniques to evaluate inequality, some Sobolev embedding. One of the most difficult points is finding the right functional spaces for the solution. Another highlight in the results is to demonstrate the convergence of the mild solution as the parameter $b$ approaches 0 .

\section{Preliminaries}

Conformable derivative model: Let the function $v:[0, \infty) \rightarrow D$, where $D$ is a Banach space. If the limitation

$$
\frac{\mathscr{C} \partial^{\beta} v(t)}{\partial t^{\beta}}:=\lim _{\varepsilon \rightarrow 0} \frac{v\left(t+\varepsilon t^{1-\beta}\right)-v(t)}{\varepsilon} \text { in } D
$$

for each $t>0$ exists, then it is called be the conformable derivative of order $\beta \in(0,1]$ of $\nu$. We can refer the reader to $[11,28]$.

Let $\mathcal{A}$ be a linear, self-adjoint, unbounded, and positive definite operator. Assume that $\mathcal{A}$ has the eigenvalues $\lambda_{n}\left(n \in \mathbb{N}^{*}\right)$ :

$$
0<\lambda_{1} \leq \lambda_{2} \leq \cdots \quad \text { with } \lambda_{n} \rightarrow \infty \text { for } n \rightarrow \infty
$$

and the corresponding eigenelements $e_{n}$ which form an orthonormal basis. For $v>0$, we introduce fractional powers of $\mathcal{A}$ as follows:

$$
D\left(\mathcal{A}^{v}\right)=\left\{g \in L^{2}(\Omega): \sum_{n=1}^{\infty}\left|\left\langle g, e_{n}\right\rangle\right|^{2} \lambda_{n}^{2 v}<\infty\right\} .
$$

The space $D\left(\mathcal{A}^{v}\right)$ is a Banach space in the following with the corresponding norm:

$$
\|g\|_{D\left(\mathcal{A}^{v}\right)}:=\left(\sum_{n=1}^{\infty}\left|\left\langle g, e_{n}\right\rangle\right|^{2} \lambda_{n}^{2 v}\right)^{\frac{1}{2}}, \quad g \in D\left(\mathcal{A}^{v}\right) .
$$

The information for negative fractional power $\mathcal{A}^{-v}$ can be defined by [29]. For any $m>0$ and a Banach space $\mathcal{B}$, we introduce the following space:

$$
C^{m}([0, T] ; \mathcal{B})=\left\{v \in C([0, T] ; \mathcal{B}): \sup _{0 \leq s<t \leq T} \frac{\|v(\cdot, t)-v(\cdot, s)\|_{\mathcal{B}}}{|t-s|^{m}}<\infty\right\}
$$

corresponding to the following norm:

$$
\|v\|_{C^{m}([0, T] ; \mathcal{B})}=\sup _{0 \leq s<t \leq T} \frac{\|v(\cdot, t)-v(\cdot, s)\|_{\mathcal{B}}}{|t-s|^{m}} .
$$

Let $0<m<1$, and we recall the following space:

$$
\mathcal{C}^{m}((0, T] ; \mathcal{B})=\left\{v \in C\left((0, T] ; L^{2}(\Omega)\right): \sup _{0<t \leq T} t^{m}\|v(\cdot, t)\|_{\mathcal{B}}<\infty\right\}
$$

with the norm $\|v\|_{\mathcal{C}^{m}((0, T] ; \mathcal{B})}:=\sup _{0<t \leq T} t^{m}\|v(\cdot, t)\|_{\mathcal{B}}$. 


\section{Inhomogeneous problem}

In this section, we consider the nonlocal value problem for equation as follows:

$$
\begin{cases}\frac{\mathscr{C}_{\partial} \beta}{\partial t^{\beta}} u(x, t)-\psi(t) \Delta u=F(x, t), & x \in \Omega, t \in(0, T), \\ u(x, t)=0, & x \in \partial \Omega, t \in(0, T), \\ a u(x, 0)+b u(x, T)=\varphi(x), & x \in \Omega,\end{cases}
$$

where $F$ is defined later.

\subsection{Existence and uniqueness of the mild solution}

In this subsection, we state the existence and uniqueness of the mild solution.

Theorem 3.1 Let $a_{0} \leq \psi(t) \leq b_{0}$, where $a_{0}$, $b_{0}$ are constants, $\varphi \in D\left(\mathcal{A}^{v+\theta}\right)$ and $F \in$ $L^{\infty}\left(0, T ; D\left(\mathcal{A}^{v+\theta}\right)\right)$, where $v>0,0<\theta<1$. Then we have the following regularity:

$$
\begin{aligned}
\|u(\cdot, t)\|_{D\left(\mathcal{A}^{v}\right)} \lesssim & t^{-\beta \theta}\left(\|\varphi\|_{D\left(\mathcal{A}^{v+\theta}\right)}+\|F\|_{L^{2 r}\left(0, T ; D\left(\mathcal{A}^{v+\theta}\right)\right)}\right) \\
& +\|F\|_{L^{\infty}\left(0, T ; D\left(\mathcal{A}^{v+\theta}\right)\right),} \quad t>0 .
\end{aligned}
$$

Proof By a simple calculation, we get the following equality:

$$
\begin{aligned}
u_{n}(t)= & \left\langle u(\cdot, t), e_{n}\right\rangle \\
= & \exp \left(-\lambda_{n} \int_{0}^{\frac{t^{\beta}}{\beta}} \psi(s) d s\right)\left\langle u(\cdot, 0), e_{n}\right\rangle \\
& +\int_{0}^{t} s^{\beta-1} \exp \left(-\lambda_{n} \int_{\frac{s^{\beta}}{\beta}}^{\frac{t^{\beta}}{\beta}} \psi(r) d r\right) F_{n}(u)(s) d s,
\end{aligned}
$$

where $F_{n}=\left\langle F, e_{n}\right\rangle$. Replacing $t$ with $T$ in the above expression, we get

$$
\begin{aligned}
u_{n}(T)= & \left\langle u(\cdot, t), e_{n}\right\rangle \\
= & \exp \left(-\lambda_{n} \int_{0}^{\frac{T^{\beta}}{\beta}} \psi(s) d s\right)\left\langle u(\cdot, 0), e_{n}\right\rangle \\
& +\int_{0}^{T} s^{\beta-1} \exp \left(-\lambda_{n} \int_{\frac{s^{\beta}}{\beta}}^{\frac{T^{\beta}}{\beta}} \psi(r) d r\right) F_{n}(u)(s) d s .
\end{aligned}
$$

The condition $a u(x, 0)+b u(x, T)=\varphi(x)$ gives the following result:

$$
\begin{aligned}
& \left(a+b \exp \left(-\lambda_{n} \int_{0}^{\frac{T^{\beta}}{\beta}} \psi(s) d s\right)\right) u_{n}(0) \\
& \quad+b \int_{0}^{T} s^{\beta-1} \exp \left(-\lambda_{n} \int_{\frac{s^{\beta}}{\beta}}^{\frac{T^{\beta}}{\beta}} \psi(r) d r\right) F_{n}(u)(s) d s=\varphi_{n} .
\end{aligned}
$$


By switching sides and combining them, we obtain the formula for $u_{n}(0)$ as follows:

$$
u_{n}(0)=\frac{\varphi_{n}-b \int_{0}^{T} s^{\beta-1} \exp \left(-\lambda_{n} \int_{\frac{s^{\beta}}{\beta}}^{\frac{T^{\beta}}{\beta}} \psi(r) d r\right) F_{n}(u)(s) d s}{a+b \exp \left(-\lambda_{n} \int_{0}^{\frac{T^{\beta}}{\beta}} \psi(s) d s\right)} .
$$

Inserting (8) into the above formula (5) and after the reduced transformation, we arrive at

$$
\begin{aligned}
u_{n}(t)= & \frac{\exp \left(-\lambda_{n} \int_{0}^{\frac{t^{\beta}}{\beta}} \psi(s) d s\right)}{a+b \exp \left(-\lambda_{n} \int_{0}^{\frac{T^{\beta}}{\beta}} \psi(s) d s\right)} \varphi_{n} \\
& -b \frac{\exp \left(-\lambda_{n} \int_{0}^{\frac{t^{\beta}}{\beta}} \psi(s) d s\right)}{a+b \exp \left(-\lambda_{n} \int_{0}^{\frac{T^{\beta}}{\beta}} \psi(s) d s\right)} \int_{0}^{T} s^{\beta-1} \exp \left(-\lambda_{n} \int_{\frac{s^{\beta}}{\beta}}^{\frac{T^{\beta}}{\beta}} \psi(r) d r\right) F_{n}(u)(s) d s \\
& +\int_{0}^{t} s^{\beta-1} \exp \left(-\lambda_{n} \int_{\frac{s^{\beta}}{\beta}}^{\frac{t^{\beta}}{\beta}} \psi(r) d r\right) F_{n}(u)(s) d s .
\end{aligned}
$$

By the properties of Fourier series, we get that

$$
\begin{aligned}
& u(x, t) \\
& =\underbrace{\sum_{n=1}^{\infty} \frac{\exp \left(-\lambda_{n} \int_{0}^{\frac{t^{\beta}}{\beta}} \psi(s) d s\right)}{a+b \exp \left(-\lambda_{n} \int_{0}^{\frac{T^{\beta}}{\beta}} \psi(s) d s\right)} \varphi_{n} e_{n}(x)}_{J_{1}(x, t)} \\
& -\underbrace{-b+b \exp \left(-\lambda_{n} \int_{0}^{\frac{T^{\beta}}{\beta}} \psi(s) d s\right)}_{n=1}\left(\int_{0}^{\left.\sum_{0}^{\infty} s^{\beta-1} \exp \left(-\lambda_{n} \int_{\frac{s^{\beta}}{\beta}}^{\frac{T^{\beta}}{\beta}} \psi(r) d r\right) F_{n}(s) d s\right) e_{n}(x)}\right. \\
& +\sum_{n=1}^{\infty}\left(\int_{0}^{t} s^{\beta-1} \exp \left(-\lambda_{n} \int_{\frac{s^{\beta}}{\beta}}^{\frac{t^{\beta}}{\beta}} \psi(r, t) d r\right) F_{n}(s) d s\right) e_{n}(x) .
\end{aligned}
$$

Using the inequality

$$
\exp \left(-\lambda_{n} \int_{0}^{\frac{t^{\beta}}{\beta}} \psi(s) d s\right) \leq \exp \left(-\lambda_{n} \frac{a_{0} t^{\beta}}{\beta}\right) \leq C_{\theta} a_{0}^{\mu} \beta^{-\theta} \lambda_{n}^{\theta} t^{-\beta \theta}
$$

we get

$$
\begin{aligned}
\left\|J_{1}(\cdot, t)\right\|_{D\left(\mathcal{A}^{v}\right)}^{2} & =\sum_{n=1}^{\infty} \lambda_{n}^{2 v}\left(\frac{\exp \left(-\lambda_{n} \int_{0}^{\frac{t^{\beta}}{\beta}} \psi(s) d s\right)}{a+b \exp \left(-\lambda_{n} \int_{0}^{\frac{T^{\beta}}{\beta}} \psi(s) d s\right)}\right)^{2} \varphi_{n}^{2} \\
& \lesssim t^{-2 \beta \theta} \sum_{n=1}^{\infty} \lambda_{n}^{2 v+2 \theta} \varphi_{n}^{2}=t^{-2 \beta \theta}\|\varphi\|_{D\left(\mathcal{A}^{v+\theta}\right)^{\cdot}}^{2}
\end{aligned}
$$


Hence, we find that

$$
\left\|J_{1}(\cdot, t)\right\|_{D\left(\mathcal{A}^{v}\right)} \lesssim t^{-\beta \theta}\|\varphi\|_{D\left(\mathcal{A}^{v+\theta}\right)}
$$

We continue to estimate $J_{2}$ as follows:

$$
\begin{aligned}
& \left\|J_{2}(\cdot, t)\right\|_{D\left(\mathcal{A}^{v}\right)}^{2} \\
& =b^{2} \sum_{n=1}^{\infty} \lambda_{n}^{2 v}\left(\frac{\exp \left(-\lambda_{n} \int_{0}^{\frac{t^{\beta}}{\beta}} \psi(s) d s\right)}{a+b \exp \left(-\lambda_{n} \int_{0}^{\frac{T^{\beta}}{\beta}} \psi(s) d s\right)}\right)^{2} \\
& \quad \times\left(\int_{0}^{T} s^{\beta-1} \exp \left(-\lambda_{n} \int_{\frac{s^{\beta}}{\beta}}^{\frac{T^{\beta}}{\beta}} \psi(r) d r\right) F_{n}(s) d s\right)^{2} \\
& \lesssim t^{-2 \beta \theta} \sum_{n=1}^{\infty} \lambda_{n}^{2 v+2 \theta}\left(\int_{0}^{T} s^{\beta-1}\left|F_{n}(s)\right|^{2} d s\right)=t^{-2 \beta \theta}\left(\int_{0}^{T} s^{\beta-1}\|F(\cdot, s)\|_{D\left(\mathcal{A}^{v+\theta}\right)}^{2} d s\right) .
\end{aligned}
$$

The Hölder inequality implies that

$$
\int_{0}^{T} s^{\beta-1}\|F(\cdot, s)\|_{D\left(\mathcal{A}^{v+\theta}\right)}^{2} d s \leq\left(\int_{0}^{T} s^{(\beta-1) r^{*} d s}\right)^{\frac{1}{r^{*}}}\left(\int_{0}^{T}\|F(\cdot, s)\|_{D\left(\mathcal{A}^{v+\theta}\right)}^{2 r} d s\right)^{1 / r}
$$

where $\frac{1}{r}+\frac{1}{r^{*}}=1$. Let us choose $r>\frac{1}{\beta}$, we find that

$$
\int_{0}^{T} s^{\beta-1}\|F(\cdot, s)\|_{D\left(\mathcal{A}^{v+\theta}\right)}^{2} d s \lesssim\|F\|_{L^{2 r}\left(0, T ; D\left(\mathcal{A}^{v+\theta}\right)\right)}^{2}
$$

Inserting the latter estimate into (13), we arrive at

$$
\left\|J_{2}(\cdot, t)\right\|_{D\left(\mathcal{A}^{v}\right)} \lesssim t^{-\beta \theta}\|F\|_{L^{2 r}\left(0, T ; D\left(\mathcal{A}^{v+\theta}\right)\right)} .
$$

The quantity $J_{3}$ is bounded by

$$
\begin{aligned}
& \left\|J_{3}(\cdot, t)\right\|_{D\left(\mathcal{A}^{v}\right)}^{2} \\
& =\sum_{n=1}^{\infty} \lambda_{n}^{2 v}\left(\int_{0}^{t} s^{\beta-1} \exp \left(-\lambda_{n} \int_{\frac{s^{\beta}}{\beta}}^{\frac{t^{\beta}}{\beta}} \psi(r) d r\right) F_{n}(s) d s\right)^{2} .
\end{aligned}
$$

It follows from the inequality

$$
\exp \left(-2 \lambda_{n} \int_{\frac{s^{\beta}}{\beta}}^{\frac{t^{\beta}}{\beta}} \psi(r) d r\right) \leq \exp \left(-\lambda_{n} \frac{a_{0}\left(t^{\beta}-s^{\beta}\right)}{\beta}\right) \leq C_{\theta} a_{0}^{\mu} \beta^{-\theta} \lambda_{n}^{\theta}\left(t^{\beta}-s^{\beta}\right)^{-\theta}
$$

for any $0 \leq s \leq t$, that

$$
\left\|J_{3}(\cdot, t)\right\|_{D\left(\mathcal{A}^{\nu}\right)}^{2} \lesssim \sum_{n=1}^{\infty} \lambda_{n}^{2 v+2 \theta}\left(\int_{0}^{t} s^{\beta-1}\left(t^{\beta}-s^{\beta}\right)^{-\theta} F_{n}^{2}(s) d s\right)
$$




$$
\begin{aligned}
& \lesssim \int_{0}^{t} s^{\beta-1}\left(t^{\beta}-s^{\beta}\right)^{-\theta}\|F(\cdot, s)\|_{D\left(\mathcal{A}^{v+\theta}\right)}^{2} d s \\
& =\|F\|_{L^{\infty}\left(0, T ; D\left(\mathcal{A}^{v+\theta}\right)\right)}^{2} \int_{0}^{t} s^{\beta-1}\left(t^{\beta}-s^{\beta}\right)^{-\theta} d s .
\end{aligned}
$$

Next, we continue to compute the integral term. Set the variable $\vartheta=s^{\beta}$. Then we get $d \vartheta=\beta s^{\beta-1} d s$. Then it follows from $0<\theta<1$ that

$$
\int_{0}^{t} s^{\beta-1}\left(t^{\beta}-s^{\beta}\right)^{-\theta} d s=\frac{1}{\beta} \int_{0}^{t^{\beta}}\left(t^{\beta}-\vartheta\right)^{-\theta} d \vartheta=\frac{t^{\beta(1-\theta)}}{\beta(1-\theta)} \leq \frac{T^{\beta(1-\theta)}}{\beta(1-\theta)} .
$$

This together with (18) yields that

$$
\left\|J_{3}(\cdot, t)\right\|_{D\left(\mathcal{A}^{v}\right)} \lesssim\|F\|_{L^{\infty}\left(0, T ; D\left(\mathcal{A}^{v+\theta}\right)\right)} .
$$

Combining (12), (16), and (20), we deduce that

$$
\begin{aligned}
& \|u(\cdot, t)\|_{D\left(\mathcal{A}^{v}\right)} \\
& \quad \leq \sum_{j=1}^{3}\left\|J_{j}(\cdot, t)\right\|_{D\left(\mathcal{A}^{v}\right)} \\
& \quad \lesssim t^{-\beta \theta}\left(\|\varphi\|_{D\left(\mathcal{A}^{v+\theta}\right)}+\|F\|_{L^{2 r}\left(0, T ; D\left(\mathcal{A}^{v+\theta}\right)\right)}\right)+\|F\|_{L^{\infty}\left(0, T ; D\left(\mathcal{A}^{v+\theta}\right)\right)}
\end{aligned}
$$

\section{The mild solution for nonlinear problem}

By the previous section, we define the following definition of a mild solution of the problem as follows.

Definition 4.1 The function $u$ is called a mild solution of the problem if $u$ belongs to the space $L^{\infty}\left(0, T ; L^{2}(\Omega)\right)$ and it also satisfies equality (1).

We recall that the formula of the solution $u$ is performed as the following form:

$$
\begin{aligned}
u(x, t)= & \sum_{n=1}^{\infty} \frac{\exp \left(-\lambda_{n} \int_{0}^{\frac{t^{\beta}}{\beta}} \psi(s) d s\right)}{a+b \exp \left(-\lambda_{n} \int_{0}^{\frac{T^{\beta}}{\beta}} \psi(s) d s\right)} \varphi_{n} e_{n}(x) \\
& -b \sum_{n=1}^{\infty} \frac{\exp \left(-\lambda_{n} \int_{0}^{\frac{t^{\beta}}{\beta}} \psi(s) d s\right)}{a+b \exp \left(-\lambda_{n} \int_{0}^{\frac{T^{\beta}}{\beta}} \psi(s) d s\right)} \\
& \times\left(\int_{0}^{T} s^{\beta-1} \exp \left(-\lambda_{n} \int_{\frac{s^{\beta}}{\beta}}^{\frac{T^{\beta}}{\beta}} \psi(r) d r\right) F_{n}(u)(s) d s\right) e_{n}(x) \\
& +\sum_{n=1}^{\infty}\left(\int_{0}^{t} s^{\beta-1} \exp \left(-\lambda_{n} \int_{\frac{s^{\beta}}{\beta}}^{\frac{t^{\beta}}{\beta}} \psi(r) d r\right) F_{n}(u)(s) d s\right) e_{n}(x) .
\end{aligned}
$$

Theorem 4.1 Let $\varphi \in L^{2}(\Omega)$, and there exists a constant $K_{f} \geq 0$ such that

$$
\left\|F\left(v_{1}\right)(\cdot, t)-F\left(v_{2}\right)(\cdot, t)\right\|_{L^{2}(\Omega)} \leq K_{f}\left\|v_{1}(\cdot, t)-v_{2}(\cdot, t)\right\|_{L^{2}(\Omega)} .
$$


If the condition $1>\left(\frac{b}{a}+1\right) \frac{K_{f} T^{\beta}}{\beta}$ is true, then problem (22) has a mild solution $u$ which belongs to the space $L^{\infty}\left(0, T ; L^{2}(\Omega)\right)$.

Proof Set the following:

$$
\mathscr{H}(u)(t)=\mathscr{H}_{0}+\mathscr{H}_{1}(u)(t)+\mathscr{H}_{2}(u)(t)
$$

where

$$
\mathscr{H}_{0}=\sum_{n=1}^{\infty} \frac{\exp \left(-\lambda_{n} \int_{0}^{\frac{t^{\beta}}{\beta}} \psi(s) d s\right)}{a+b \exp \left(-\lambda_{n} \int_{0}^{\frac{T^{\beta}}{\beta}} \psi(s) d s\right)} \varphi_{n} e_{n}(x)
$$

and

$$
\begin{aligned}
\mathscr{H}_{1}(u)= & -b \sum_{n=1}^{\infty} \frac{\exp \left(-\lambda_{n} \int_{0}^{\frac{t^{\beta}}{\beta}} \psi(s) d s\right)}{a+b \exp \left(-\lambda_{n} \int_{0}^{\frac{T^{\beta}}{\beta}} \psi(s) d s\right)} \\
& \times\left(\int_{0}^{T} s^{\beta-1} \exp \left(-\lambda_{n} \int_{\frac{s^{\beta}}{\beta}}^{\frac{T^{\beta}}{\beta}} \psi(r) d r\right) F_{n}(u)(s) d s\right) e_{n}(x)
\end{aligned}
$$

and

$$
\mathscr{H}_{2, \beta}(u)(t)=\sum_{n=1}^{\infty}\left(\int_{0}^{t} s^{\beta-1} \exp \left(-\lambda_{n} \int_{\frac{s^{\beta}}{\beta}}^{\frac{t^{\beta}}{\beta}} \psi(r) d r\right) F_{n}(u)(s) d s\right) e_{n}(x) .
$$

We have to show that the equation $\mathscr{H} u=u$ has a unique solution. Applying the Hölder inequality, we find that

$$
\begin{aligned}
\| & \mathscr{H}_{2, \beta}(u)(t)-\mathscr{H}_{2, \beta}(v)(t) \|_{L^{2}(\Omega)}^{2} \\
& =\sum_{n=1}^{\infty}\left[\int_{0}^{t} s^{\beta-1} \exp \left(-\lambda_{n} \int_{\frac{s^{\beta}}{\beta}}^{\frac{t^{\beta}}{\beta}} \psi(r) d r\right)\left(F_{n}(u)(s)-F_{n}(v)(s)\right) d s\right]^{2} \\
& \leq \sum_{n=1}^{\infty}\left[\int_{0}^{t} s^{\beta-1} d s\right]\left[\int_{0}^{t} s^{\beta-1} \exp \left(-2 \lambda_{n} \int_{\frac{s^{\beta}}{\beta}}^{\frac{t^{\beta}}{\beta}} \psi(r) d r\right)\left(F_{n}(u)(s)-F_{n}(v)(s)\right)^{2} d s\right] \\
& \leq \frac{T^{\beta}}{\beta}\left[\int_{0}^{t} s^{\beta-1}\|F(u)(\cdot, s)-F(v)(\cdot, s)\|_{L^{2}(\Omega)}^{2} d s\right] \\
& \leq \frac{T^{\beta}}{\beta} K_{f}^{2}\|u-v\|_{L^{\infty}\left(0, T ; L^{2}(\Omega)\right)}^{2}\left[\int_{0}^{t} s^{\beta-1} d s\right]=\frac{K_{f}^{2} T^{2 \beta}}{\beta^{2}}\|u-v\|_{L^{\infty}\left(0, T ; L^{2}(\Omega)\right)}^{2},
\end{aligned}
$$

and using the inequality

$$
\left|-b \frac{\exp \left(-\lambda_{n} \int_{0}^{\frac{t^{\beta}}{\beta}} \psi(s) d s\right)}{a+b \exp \left(-\lambda_{n} \int_{0}^{\frac{T^{\beta}}{\beta}} \psi(s) d s\right)}\right| \leq \frac{b}{a}
$$


we get that

$$
\begin{aligned}
\| & \mathscr{H}_{1, \beta}(u)(t)-\mathscr{H}_{1, \beta}(v)(t) \|_{L^{2}(\Omega)}^{2} \\
& \leq \frac{b^{2}}{a^{2}} \sum_{n=1}^{\infty}\left(\int_{0}^{T} s^{\beta-1} \exp \left(-\lambda_{n} \int_{\frac{s^{\beta}}{\beta}}^{\frac{T^{\beta}}{\beta}} \psi(r) d r\right)\left(F_{n}(u)(s)-F_{n}(v)(s)\right) d s\right)^{2} \\
& \leq \frac{b^{2}}{a^{2}} \sum_{n=1}^{\infty}\left[\int_{0}^{t} s^{\beta-1} d s\right]\left(\int_{0}^{T} s^{\beta-1} \exp \left(-2 \lambda_{n} \int_{\frac{s^{\beta}}{\beta}}^{\frac{T^{\beta}}{\beta}} \psi(r) d r\right)\left(F_{n}(u)(s)-F_{n}(v)(s)\right)^{2} d s\right) \\
& \leq \frac{b^{2}}{a^{2}} \frac{T^{\beta}}{\beta}\left(\int_{0}^{T} s^{\beta-1}\left(F_{n}(u)(s)-F_{n}(v)(s)\right)^{2} d s\right) \leq \frac{b^{2}}{a^{2}} \frac{K_{f}^{2} T^{2 \beta}}{\beta^{2}}\|u-v\|_{L^{\infty}\left(0, T ; L^{2}(\Omega)\right)^{\circ}}^{2} \quad \text { (28) }
\end{aligned}
$$

Combining (27) and (28) leads to

$$
\begin{aligned}
& \left\|\mathscr{H}_{\beta}(u)(t)-\mathscr{H}_{\beta}(v)(t)\right\|_{L^{2}(\Omega)} \\
& \quad=\left\|\mathscr{H}_{1, \beta}(u)(t)-\mathscr{H}_{1, \beta}(v)(t)\right\|_{L^{2}(\Omega)}+\left\|\mathscr{H}_{2, \beta}(u)(t)-\mathscr{H}_{2, \beta}(v)(t)\right\|_{L^{2}(\Omega)} \\
& \quad \leq \frac{K_{f} T^{\beta}}{\beta}\left(1+\frac{b}{a}\right)\|u-v\|_{L^{\infty}\left(0, T ; L^{2}(\Omega)\right)} .
\end{aligned}
$$

Since the left-hand side of the above observation is independent of $t$, we deduce that

$$
\left\|\mathscr{H}_{\beta}(u)-\mathscr{H}_{\beta}(v)\right\|_{L^{\infty}\left(0, T ; L^{2}(\Omega)\right)} \leq \frac{K_{f} T^{\beta}}{\beta}\left(1+\frac{b}{a}\right)\|u-v\|_{L^{\infty}\left(0, T ; L^{2}(\Omega)\right)} .
$$

Let us choose $T$ such that

$$
T \leq\left(\frac{\beta a}{(a+b) K_{f}}\right)^{1 / \beta}
$$

we know that $\mathscr{H}$ is a contraction mapping. Next, we continue to show that if $v \in$ $L^{\infty}\left(0, T ; L^{2}(\Omega)\right)$, then $\mathscr{H}_{\beta}(v) \in L^{\infty}\left(0, T ; L^{2}(\Omega)\right)$. We only check that if $v=0$, then

$$
\mathscr{H}_{\beta}(v)(\cdot, t)=\sum_{n=1}^{\infty} \frac{\exp \left(-\lambda_{n} \int_{0}^{\frac{t^{\beta}}{\beta}} \psi(s) d s\right)}{a+b \exp \left(-\lambda_{n} \int_{0}^{\frac{T^{\beta}}{\beta}} \psi(s) d s\right)} \varphi_{n} e_{n}(x),
$$

which allows us to obtain that

$$
\begin{aligned}
\left\|\mathscr{H}_{\beta}(v)(\cdot, t)\right\|_{L^{2}(\Omega)}^{2} & =\sum_{n=1}^{\infty}\left(\frac{\exp \left(-\lambda_{n} \int_{0}^{\frac{t^{\beta}}{\beta}} \psi(s) d s\right)}{a+b \exp \left(-\lambda_{n} \int_{0}^{\frac{T^{\beta}}{\beta}} \psi(s) d s\right)}\right)^{2} \varphi_{n}^{2} \\
& \leq \frac{1}{a^{2}} \sum_{n=1}^{\infty} \varphi_{n}^{2} \\
& =\frac{1}{a^{2}}\|\varphi\|_{L^{2}(\Omega)}^{2} .
\end{aligned}
$$


The latter estimate leads to $\mathscr{H}_{\beta}: L^{\infty}\left(0, T ; L^{2}(\Omega)\right) \rightarrow L^{\infty}\left(0, T ; L^{2}(\Omega)\right)$. By applying the Banach fixed point theory, we can deduce that our problem (1) has a unique solution $u \in$ $L^{\infty}\left(0, T ; L^{2}(\Omega)\right)$.

Theorem 4.2 Let $w_{a}$ be the solution of the initial value problem

$$
\begin{cases}\frac{\mathscr{C}_{\partial} \beta}{\partial t^{\beta}} u(x, t)-\psi(t) \Delta u=F(x, t), & x \in \Omega, t \in(0, T), \\ u(x, t)=0, & x \in \partial \Omega, t \in(0, T), \\ a u(x, 0)=\varphi(x), & x \in \Omega .\end{cases}
$$

Then we have

$$
\lim _{b \rightarrow 0}\left\|w_{a, \beta}-u_{a, b, \beta}\right\|_{L^{\infty}\left(0, T ; L^{2}(\Omega)\right)}=0
$$

and

$$
\left\|w_{a, \beta}-u_{a, b, \beta}\right\|_{L^{\infty}\left(0, T ; L^{2}(\Omega)\right)} \leq \frac{b\|\varphi\|_{L^{2}(\Omega)}}{a^{2}\left(1-\left(\frac{b}{a}+1\right) \frac{K_{f} T^{\beta}}{\beta}\right)} .
$$

Proof It is easy to see that the mild solution of problem (33) is given by

$$
\begin{aligned}
w_{a, \beta}(x, t) & \\
= & \sum_{n=1}^{\infty} \frac{\exp \left(-\lambda_{n} \int_{0}^{\frac{t^{\beta}}{\beta}} \psi(s) d s\right)}{a} \varphi_{n} e_{n}(x) \\
& -b \sum_{n=1}^{\infty} \frac{\exp \left(-\lambda_{n} \int_{0}^{\frac{t^{\beta}}{\beta}} \psi(s) d s\right)}{a} \\
& \times\left(\int_{0}^{T} s^{\beta-1} \exp \left(-\lambda_{n} \int_{\frac{s^{\beta}}{\beta}}^{\frac{T^{\beta}}{\beta}} \psi(r) d r\right) F_{n}\left(w_{a, \beta}\right)(s) d s\right) e_{n}(x) \\
& +\sum_{n=1}^{\infty}\left(\int_{0}^{t} s^{\beta-1} \exp \left(-\lambda_{n} \int_{\frac{s^{\beta}}{\beta}}^{\frac{t^{\beta}}{\beta}} \psi(r) d r\right) F_{n}\left(w_{a, \beta}\right)(s) d s\right) e_{n}(x) .
\end{aligned}
$$

This together with (22) yields

$$
\begin{aligned}
& u_{a, b, \beta}(x, t)-w_{a, \beta}(x, t) \\
& =\sum_{n=1}^{\infty}\left(\frac{\exp \left(-\lambda_{n} \int_{0}^{\frac{t^{\beta}}{\beta}} \psi(s) d s\right)}{a}-\frac{\exp \left(-\lambda_{n} \int_{0}^{\frac{t^{\beta}}{\beta}} \psi(s) d s\right)}{a+b \exp \left(-\lambda_{n} \int_{0}^{\frac{T^{\beta}}{\beta}} \psi(s) d s\right)}\right) \varphi_{n} e_{n}(x) \\
& \quad-b \sum_{n=1}^{\infty} \frac{\exp \left(-\lambda_{n} \int_{0}^{\frac{t^{\beta}}{\beta}} \psi(s) d s\right)}{a} \\
& \quad \times\left[\int_{0}^{T} s^{\beta-1} \exp \left(-\lambda_{n} \int_{\frac{s^{\beta}}{\beta}}^{\frac{T^{\beta}}{\beta}} \psi(r) d r\right)\left(F_{n}\left(w_{a, \beta}\right)(s)-F_{n}\left(u_{a, b, \beta}\right)(s)\right) d s\right] e_{n}(x)
\end{aligned}
$$




$$
\begin{aligned}
& +\sum_{n=1}^{\infty}\left[\int_{0}^{t} s^{\beta-1} \exp \left(-\lambda_{n} \int_{\frac{s}{\beta}}^{\frac{t^{\beta}}{\beta}} \psi(r) d r\right)\left(F_{n}\left(w_{a, \beta}\right)(s)-F_{n}\left(u_{a, b, \beta}\right)(s)\right) d s\right] e_{n}(x) \\
= & A_{1}+A_{2}+A_{3} .
\end{aligned}
$$

The term $A_{1}$ is bounded by

$$
\begin{aligned}
\left\|A_{1}\right\|_{L^{2}(\Omega)}^{2} & =\frac{b^{2}}{a^{2}} \sum_{n=1}^{\infty}\left(\frac{\exp \left(-\lambda_{n} \int_{0}^{\frac{t^{\beta}}{\beta}} \psi(s) d s\right) \exp \left(-\lambda_{n} \int_{0}^{\frac{T^{\beta}}{\beta}} \psi(s) d s\right)}{a+b \exp \left(-\lambda_{n} \int_{0}^{\frac{T^{\beta}}{\beta}} \psi(s) d s\right)}\right)^{2} \varphi_{n}^{2} \\
& \leq \frac{b^{2}}{a^{4}} \sum_{n=1}^{\infty} \varphi_{n}^{2}=\frac{b^{2}}{a^{4}}\|\varphi\|_{L^{2}(\Omega)}^{2} .
\end{aligned}
$$

Using $\exp \left(-2 \lambda_{n} \int_{0}^{\frac{t^{\beta}}{\beta}} \psi(s) d s\right) \leq 1$, the term $A_{2}$ is bounded by

$$
\begin{aligned}
\left\|A_{2}\right\|_{L^{2}(\Omega)}^{2} & =\frac{b^{2}}{a^{2}} \sum_{n=1}^{\infty}\left[\int_{0}^{T} s^{\beta-1} \exp \left(-\lambda_{n} \int_{\frac{s^{\beta}}{\beta}}^{\frac{T^{\beta}}{\beta}} \psi(r) d r\right)\left(F_{n}\left(w_{a, \beta}\right)(s)-F_{n}\left(u_{a, b, \beta}\right)\right)(s) d s\right]^{2} \\
\leq & \frac{b^{2}}{a^{2}} \sum_{n=1}^{\infty}\left(\int_{0}^{T} s^{\beta-1} d s\right) \\
& \times \int_{0}^{T} s^{\beta-1} \exp \left(-2 \lambda_{n} \int_{\frac{s^{\beta}}{\beta}}^{\frac{T^{\beta}}{\beta}} \psi(r) d r\right)\left(F_{n}\left(w_{a, \beta}\right)(s)-F_{n}\left(u_{a, b, \beta}\right)(s)\right)^{2} d s \\
\leq & \frac{b^{2}}{a^{2}} \frac{T^{\beta}}{\beta}\left[\int_{0}^{t} s^{\beta-1}\left\|F\left(w_{a, \beta}\right)(\cdot, s)-F\left(u_{a, b, \beta}\right)(\cdot, s)\right\|_{L^{2}(\Omega)}^{2} d s\right] \\
\leq & \frac{b^{2}}{a^{2}} \frac{K_{f}^{2} T^{2 \beta}}{\beta^{2}}\left\|w_{a, \beta}-u_{a, b, \beta}\right\|_{L^{\infty}\left(0, T ; L^{2}(\Omega)\right)}^{2} .
\end{aligned}
$$

The term $A_{3}$ can be estimated as follows:

$$
\begin{aligned}
\left\|A_{3}\right\|_{L^{2}(\Omega)}^{2} & =\sum_{n=1}^{\infty}\left[\int_{0}^{t} s^{\beta-1} \exp \left(-\lambda_{n} \int_{\frac{s^{\beta}}{\beta}}^{\frac{t^{\beta}}{\beta}} \psi(r) d r\right)\left(F_{n}\left(w_{a, \beta}\right)(s)-F_{n}\left(u_{a, b, \beta}(s)\right) d s\right]^{2}\right. \\
& \leq \sum_{n=1}^{\infty}\left[\int_{0}^{T} s^{\beta-1} d s\right]\left[\int_{0}^{t} s^{\beta-1}\left\|F\left(w_{a, \beta}\right)(\cdot, s)-F\left(u_{a, b, \beta}\right)(\cdot, s)\right\|_{L^{2}(\Omega)}^{2} d s\right] \\
& \leq \frac{K_{f}^{2} T^{2 \beta}}{\beta^{2}}\left\|w_{a, \beta}-u_{a, b, \beta}\right\|_{L^{\infty}\left(0, T ; L^{2}(\Omega)\right)}^{2} .
\end{aligned}
$$

Therefore, we find that

$$
\left\|A_{3}\right\|_{L^{2}(\Omega)} \leq \frac{K_{f} T^{\beta}}{\beta}\left\|w_{a, \beta}-u_{a, b, \beta}\right\|_{L^{\infty}\left(0, T ; L^{2}(\Omega)\right)}
$$




\section{Some above observations lead to}

$$
\begin{aligned}
& \left\|w_{a, \beta}-u_{a, b, \beta}\right\|_{L^{\infty}\left(0, T ; L^{2}(\Omega)\right)} \\
& \quad \leq\left\|A_{1}\right\|_{L^{2}(\Omega)}+\left\|A_{2}\right\|_{L^{2}(\Omega)}+\left\|A_{3}\right\|_{L^{2}(\Omega)} \\
& \quad \leq \frac{b}{a^{2}}\|\varphi\|_{L^{2}(\Omega)}+\left(\frac{b}{a}+1\right) \frac{K_{f} T^{\beta}}{\beta}\left\|w_{a, \beta}-u_{a, b, \beta}\right\|_{L^{\infty}\left(0, T ; L^{2}(\Omega)\right)} .
\end{aligned}
$$

Hence, we find that

$$
\left\|w_{a, \beta}-u_{a, b, \beta}\right\|_{L^{\infty}\left(0, T ; L^{2}(\Omega)\right)} \leq \frac{b\|\varphi\|_{L^{2}(\Omega)}}{a^{2}\left(1-\left(\frac{b}{a}+1\right) \frac{K_{f} T^{\beta}}{\beta}\right)} .
$$

It is easy to see that

$$
\lim _{b \rightarrow 0}\left\|w_{a, \beta}-u_{a, b, \beta}\right\|_{L^{\infty}\left(0, T ; L^{2}(\Omega)\right)}=0 .
$$

\section{Acknowledgements}

This research is supported by Industrial University of Ho Chi Minh City (IUH) under grant number 66/HĐ-ĐHCN. The authors would like to thank the reviewers and editor for their constructive comments and valuable suggestions that improved the quality of this paper.

\section{Funding}

Not applicable.

\section{Availability of data and materials}

Not applicable.

\section{Competing interests}

The authors declare that they have no competing interests.

\section{Authors' contributions}

The authors declare that the study was realized in collaboration with the same responsibility. All authors contributed equally to the writing of this paper. All authors read and approved the final manuscript.

\section{Author details}

${ }^{1}$ Faculty of Fundamental Science, Industrial University of Ho Chi Minh City, Ho Chi Minh City, Vietnam. ${ }^{2}$ Division of Applied Mathematics, Thu Dau Mot University, Binh Duong Province, Vietnam. ${ }^{3}$ Faculty of Information Technology, Ho Chi Minh City University of Technology, Ho Chi Minh City, Vietnam.

\section{Publisher's Note}

Springer Nature remains neutral with regard to jurisdictional claims in published maps and institutional affiliations.

Received: 12 March 2021 Accepted: 7 April 2021 Published online: 17 April 2021

\section{References}

1. Chung, W.S.: Fractional Newton mechanics with conformable fractional derivative. J. Comput. Appl. Math. 290, 150-158 (2015)

2. Morales-Delgado, V.F., Gómez-Aguilar, J.F., Escobar-Jiménez, R.F., Taneco-Hernández, M.A.: Fractional conformable derivatives of Liouville-Caputo type with low-fractionality. Phys. A, Stat. Mech. Appl. 503, 424-438 (2018)

3. He, S., Sun, K., Mei, X., Yan, B., Xu, S.: Numerical analysis of a fractional-order chaotic system based on conformable fractional-order derivative. Eur. Phys. J. Plus 132, 36 (2017)

4. Anderson, R., Ulness, J.D.: Properties of the Katugampola fractional derivative with potential application in quantum mechanics. J. Math. Phys. 6, 063502 (2015) 18 pp

5. Tuan, N.H., Ngoc, T.B., Baleanu, D., O'Regan, D.: On well-posedness of the sub-diffusion equation with conformable derivative model. Commun. Nonlinear Sci. Numer. Simul. 89, 105332 (2020)

6. Alharbi, F.M., Baleanu, D., Ebaid, A.: Physical properties of the projectile motion using the conformable derivative. Chin. J. Phys. 58, 18-28 (2019)

7. Baleanu, D., Fernandez, A.: On some new properties of fractional derivatives with Mittag-Leffler kernel. Commun. Nonlinear Sci. Numer. Simul. 59, 444-462 (2018)

8. Baleanu, D., Fernandez, A., Fokas, A.S.: Solving PDEs of fractional order using the unified transform method. Appl. Math. Comput. 339, 738-749 (2018) 
9. Baleanu, D., Mousalou, A., Rezapour, S.: A new method for investigating approximate solutions of some fractional integro-differential equations involving the Caputo-Fabrizio derivative. Adv. Differ. Equ. 2017, Paper No. 51 (2017)

10. Aphithana, A., Ntouyas, S.K., Tariboon, J.: Forced oscillation of fractional differential equations via conformable derivatives with damping term. Bound. Value Probl. 2019, 47 (2019)

11. Atangana, A., Baleanu, D., Alsaedi, A.: New properties of conformable derivative. Open Math. 13, $889-898$ (2015)

12. Çenesiz, Y., Baleanu, D., Kurt, A., Tasbozan, O.: New exact solutions of Burgers' type equations with conformable derivative. Waves Random Complex Media 27(1), 103-116 (2017)

13. Karapinar, E., Binh, H.D., Luc, N.H., Can, N.H.: On continuity of the fractional derivative of the time-fractional semilinear pseudo-parabolic systems. Adv. Differ. Equ. 2021(1), 70, 1-24 (2021)

14. Tuan, N.H., Thach, T.N., Can, N.H., O'Regan, D.: Regularization of a multidimensional diffusion equation with conformable time derivative and discrete data. Math. Methods Appl. Sci. 44(4), 2879-2891 (2021)

15. Binh, T.T., Luc, N.H., O'Regan, D., Can, N.H.: On an initial inverse problem for a diffusion equation with a conformable derivative. Adv. Differ. Equ. 2019(1), 481, 1-24 (2019)

16. Tuan, N.H., Zhou, Y., Can, N.H.: Identifying inverse source for fractional diffusion equation with Riemann-Liouville derivative. Comput. Appl. Math. 39(2), 1-16 (2020)

17. Tuan, N.H., Baleanu, D., Thach, T.N., O'Regan, D., Can, N.H.: Approximate solution for a 2-D fractional differential equation with discrete random noise. Chaos Solitons Fractals 133, 109650 (2020)

18. Tuan, N.H., Huynh, L.N., Baleanu, D., Can, N.H.: On a terminal value problem for a generalization of the fractional diffusion equation with hyper-Bessel operator. Math. Methods Appl. Sci. 43(6), 2858-2882 (2020)

19. Luc, N.H., Baleanu, D., Long, L.D., Can, N.-H.: Reconstructing the right-hand side of a fractional subdiffusion equation from the final data. J. Inequal. Appl. 2020, 53 (2020)

20. Tuan, N.H., Baleanu, D., Thach, T.N., O'Regan, D., Can, N.H.: Final value problem for nonlinear time fractional reaction-diffusion equation with discrete data. J. Comput. Appl. Math. 376, 112883 (2020)

21. Triet, A.N., O'Regan, D., Baleanu, D., Luc, N.H., Can, N.: A filter method for inverse nonlinear sideways heat equation Adv. Differ. Equ. 2020, 149 (2020)

22. Afshari, H., Kalantari, S., Karapinar, E.: Solution of fractional differential equations via coupled fixed point. Electron. J. Differ. Equ. 2015, 286 (2015)

23. Afshari, H., Karapınar, E.: A discussion on the existence of positive solutions of the boundary value problems via $\psi$-Hilfer fractional derivative on b-metric spaces. Adv. Differ. Equ. 2020(1), 1 (2020)

24. Sevinik Adigüzel, R., Aksoy, Ü., Karapinar, E., Erhan, I.M.: On the solution of a boundary value problem associated with a fractional differential equation. Math. Methods Appl. Sci. (2020). https://doi.org/10.1002/mma.6652

25. Patil, J., Chaudhari, A., Mohammed, A.B.D.O., Hardan, B.: Upper and lower solution method for positive solution of generalized Caputo fractional differential equations. Adv. Theor. Nonlinear Anal. Appl. 4(4), 279-291 (2020)

26. Baitiche, Z., Derbazi, C., Benchohra, M.: $\psi$-Caputo fractional differential equations with multi-point boundary conditions by topological degree theory. Results Nonlinear Anal. 3(4), 166-178 (2020)

27. Kandasamy, M., Annamalai, A.: Existence results for fractional integrodifferential systems with interval impulse via sectorial operator. Results Nonlinear Anal. 2(4), 169-181 (2019)

28. Khalil, R., Al Horani, M., Yousef, A., Sababheh, M.: A new definition of fractional derivative. J. Comput. Appl. Math. 264, $65-70$ (2014)

29. Brezis, H.: Functional Analysis, Sobolev Spaces and Partial Differential Equations. Springer, New York (2011)

\section{Submit your manuscript to a SpringerOpen ${ }^{\circ}$ journal and benefit from:}

- Convenient online submission

- Rigorous peer review

- Open access: articles freely available online

- High visibility within the field

- Retaining the copyright to your article

Submit your next manuscript at $>$ springeropen.com 